\title{
Tasas de interés activas y política monetaria en el Perú. Un análisis con datos de bancos individuales
}

\author{
Rodolfo Cermeño, Oscar Dancourt, \\ Gustavo Ganiko y Waldo Mendoza ${ }^{* *}$
}

\begin{abstract}
RESUMEN
Este trabajo evalúa empíricamente el canal de tasas de interés en el mecanismo de transmisión de la política monetaria en el Perú, durante el periodo junio 2003-junio 2010, empleando datos mensuales de bancos individuales. Se estudian los dos principales instrumentos de política utilizados bajo el régimen de metas de inflación: la tasa de política monetaria y la tasa de encaje. Utilizando un modelo de datos de panel dinámico, nuestro trabajo tiene dos resultados básicos. En primer lugar, un alza de la tasa de interés de referencia tiene un impacto positivo y significativo sobre las tasas de interés de los préstamos comerciales fijadas por los seis bancos más grandes del país. En segundo lugar, no encontramos evidencia que sugiera que la tasa de encaje a los depósitos en moneda nacional influye sobre estas mismas tasas de interés fijadas por estos seis bancos durante el periodo analizado.
\end{abstract}

Palabras clave: mecanismo de transmisión de la política monetaria, canal de tasas de interés, tasa de interés de referencia, tasa de encaje, modelo Bernanke-Blinder.

Clasificación JEL: E44; E52

\footnotetext{
* Documento que forma parte del proyecto «Instrumentos y reglas de política monetaria en una economía semi-dolarizada: evaluación empírica del caso peruano», del grupo Macroeconomía Aplicada, con el apoyo financiero del Vicerrectorado de Investigación de la Pontificia Universidad Católica del Perú (PUCP). Una primera versión de este artículo apareció como documento de trabajo del Departamento de Economía (DD410).

** Rodolfo Cermeño es profesor e investigador del Centro de Investigación y Docencia Económicas (CIDE, México), Oscar Dancourt y Waldo Mendoza son profesores e investigadores del Departamento de Economía de la PUCP (DE-PUCP) y Gustavo Ganiko es coordinador en la Dirección Académica de Planeamiento y Evaluación de la PUCP (DAPE-PUCP).
} 


\title{
Active Interest Rates and Monetary Policy: An Analysis with Individual Banks Data
}

\begin{abstract}
This paper investigates empirically the interest rate channel of the transmission mechanism of the monetary policy in Peru. Using monthly data for the six largest banks for the period June $2003-$ June 2010 we study the two main policy instruments used under the inflation-target regime: the rate of monetary policy and the required bank reserves rate. We fit a dynamic panel data model obtaining two fundamental results. First, increases in the rate of monetary policy affect positively and significantly the interest rates on commercial loans charged by the six largest banks of the country. Second, no evidence is found that the required bank reserves rate on deposits in Peruvian currency influences the same interest rates charged by the six largest banks during the period considered.
\end{abstract}

Keywords: transmission mechanism of monetary policy, interest rate cannel, rate of monetary policy, required bank reserves rate, Bernanke-Blinder model.

JEL Codes: E44; E52

\section{INTRODUCCIÓN}

Si dejamos de lado la intervención en el mercado cambiario, la tasa de encaje para los depósitos bancarios en moneda nacional $(\mathrm{MN})$ y la tasa de interés de referencia para el mercado interbancario, donde los bancos se prestan fondos entre sí, han sido los dos principales instrumentos de la política monetaria aplicada en el pasado reciente por el Banco Central de Reserva del Perú (BCRP) en el marco de un sistema de metas de inflación.

El BCRP elevó la tasa de referencia y la tasa de encaje durante el primer semestre de 2008, cuando la inflación sobrepasó el rango meta del 3\% anual y la economía peruana crecía a un ritmo del $10 \%$ anual; luego, redujo ambas tasas, entre fines de 2008 y principios de 2009, cuando la actividad económica sufrió los primeros embates recesivos de la crisis financiera global. Posteriormente, volvió a subir la tasa de interés de referencia y la tasa de encaje en MN a partir de 2010 cuando la economía peruana se reactivó vigorosamente apoyada en una notable recuperación de los precios internacionales de los minerales. Cabe anotar, sin embargo, que el BCRP no respondió con un agresivo ciclo de rebajas de la tasa de interés de referencia al rápido enfriamiento de la economía peruana ocurrido durante 2013-2014, causado por una caída de los precios mundiales de los metales y una salida de capitales vinculada al ajuste de la política monetaria norteamericana. El BCRP se limitó a reducir progresivamente la tasa de encaje en MN desde 2013 mientras mantuvo casi constante la tasa de interés de referencia hasta mediados de 2014. 
En un contexto donde la autoridad monetaria busca estabilizar el nivel de precios alrededor de un cierto nivel meta, y el sistema financiero está dominado por los bancos, el mecanismo de transmisión que conecta en el corto plazo estos dos instrumentos de política (la tasa de interés de referencia y la tasa de encaje en $\mathrm{MN}$ ) con el nivel de precios consta de cuatro eslabones básicos. El primer eslabón es el que conecta los instrumentos de la política monetaria con las tasas de interés (activas) cobradas por los bancos y con los volúmenes prestados a firmas y familias. Si el banco central sube, por ejemplo, la tasa de interés de referencia o la tasa de encaje en $\mathrm{MN}$, se espera que esto eleve la tasa de interés activa en $\mathrm{MN}$ y/o que reduzca el crédito bancario en $\mathrm{MN}$.

El segundo eslabón conecta el gasto de consumo e inversión del sector privado con el costo y la disponibilidad de crédito. El gasto agregado del sector privado depende inversamente de la tasa activa de interés y directamente de los volúmenes prestados ${ }^{1}$. El tercer eslabón establece que la actividad económica y el empleo total en la economía dependen del gasto agregado del sector privado (consumo más inversión). El cuarto y último eslabón establece que el nivel de precios depende directamente de la actividad económica, si consideramos dada la capacidad productiva de la economía o producto potencial.

De esta manera, un alza de la tasa de interés de referencia o un alza de la tasa de encaje encarecen y hacen menos abundante el crédito otorgado al sector privado por el sistema bancario. Disminuye entonces el gasto agregado del sector privado, lo que hace caer la actividad económica y el empleo agregado y, finalmente, el aumento de la capacidad ociosa y del desempleo reducen el nivel de precios ${ }^{2}$.

Si se acepta esta apretada descripción del mecanismo de transmisión de la política monetaria vía los bancos comerciales, es claro que el primer eslabón de esta cadena causal es crucial. Si ese eslabón se quiebra no habría conexión, a través de este canal del crédito $^{3}$, entre los instrumentos y los objetivos de la política monetaria, sean estos la estabilidad del nivel de precios y/o el pleno empleo.

¿Qué tan efectivo ha sido el uso de la tasa de encaje en comparación con el de la tasa de referencia durante el periodo 2003-2010 ${ }^{4}$ ? Esta es la pregunta que este texto intenta responder, tomando en cuenta que dicho periodo abarca dos fases de salida de una recesión (2003-2005 y 2009-2010), un auge (2006-2008) sin precedentes en la economía peruana, y una recesión (2008-2009) donde se aplicó por vez primera una política monetaria expansiva. Se considera aquí que ambos instrumentos de política monetaria son efectivos si impactan sobre las tasas de interés en MN fijadas para los préstamos

\footnotetext{
Esto incluye el caso en que existe racionamiento en los mercados de crédito; si no existe racionamiento, la demanda agregada solo depende de las tasas de interés. Véase Stiglitz y Greenwald (2003, Cap. 6).

2 Se supone que la inflación esperada es nula.

3 El canal del crédito en sentido amplio se refiere aquí al efecto que tienen los instrumentos de la política monetaria sobre los volúmenes prestados y las tasas de interés cobradas por los bancos; véase Bernanke y Gertler (1995).

4 No analizamos el periodo 2011-2014 porque habría que reconstruir las series de las tasas activas de los préstamos comerciales para hacerlas compatibles con las del periodo 2003-2010.
} 
comerciales ${ }^{5}$ por los seis bancos más grandes del país. Los préstamos comerciales de estos seis bancos representan más de dos terceras partes del crédito del sistema bancario al sector privado en moneda nacional durante 2003-2010.

Lahura (2006) y el Reporte de Inflación de junio de 2009 (BCRP, 2009) demuestran que este eslabón existe, cuando el instrumento de política es la tasa de interés de referencia para el mercado interbancario. Utilizando datos agregados de las tasas de interés bancarias activas y pasivas, Lahura (2006) muestra que este eslabón se crea a principios de los años 2000, cuando el BCRP transita de un régimen basado en el control de un agregado monetario a otro basado en el control de la tasa de interés del mercado interbancario, donde los bancos comerciales típicamente se prestan entre sí fondos por un día.

Este primer eslabón es el que queremos evaluar empíricamente en este texto, utilizando datos de bancos individuales, y limitando la pesquisa al impacto que ambos instrumentos de la política monetaria (tasa de referencia y tasa de encaje en $\mathrm{MN}$ ) tienen sobre las tasas de interés activas en MN fijadas por los seis bancos más grandes del país ${ }^{6}$.

Tres trabajos importantes que utilizan un modelo de panel dinámico para determinar el impacto de la tasa de interés de referencia sobre las tasas de interés activas y pasivas fijadas por distintos bancos, son el de Weth (2002) para Alemania, el de Gambacorta (2004) para Italia, y el de Bernstein y Fuentes (2003) para Chile. Estos trabajos encuentran que la tasa de interés de referencia incide de la manera esperada sobre las tasas activas fijadas por los bancos comerciales; algunos de ellos controlan por el efecto que puedan tener otras variables macroeconómicas o microeconómicas. Nuestro modelo empírico está basado en Weth (2002).

Nuestro trabajo tiene dos resultados básicos. En primer lugar, encontramos que un alza de la tasa de interés de referencia tiene un impacto positivo y estadísticamente significativo sobre las tasas de interés de los préstamos comerciales de 0 a 360 días fijadas por los seis bancos más grandes del país durante el periodo junio de 2003-junio de 2010. En segundo lugar, no encontramos evidencia que sugiera que la tasa de encaje a los depósitos en $\mathrm{MN}$ influye de alguna manera sobre estas mismas tasas de interés fijadas por estos seis bancos durante el mismo periodo.

Este documento consta de cinco secciones, incluyendo esta introducción. En la sección 2 se presenta el marco teórico. En la sección 3 se describe el procedimiento econométrico aplicado en la investigación. En la sección 4 se presentan los principales resultados encontrados. La sección 5 contiene las conclusiones.

\footnotetext{
5 Los préstamos comerciales son los préstamos a las empresas excluyendo a las muy pequeñas (microempresas).

6 Alternativamente, se puede medir el impacto que la tasa de referencia o la tasa de encaje tienen sobre el volumen de crédito en MN otorgado por bancos individuales; véase Kashyap y Stein (1995, 2000) y Ehrman et al. (2001). Para el caso brasileño, véase Takeda, Rocha y Nakane (2005) y, para el caso peruano, véase Dancourt (2012).
} 


\section{EL MARCO TEÓRICO}

El marco conceptual de este texto es el modelo Bernanke-Blinder (1988) que es un modelo IS-LM con bancos. El rasgo especifico de este modelo IS-LM ampliado es que el sistema bancario y el mercado de bonos compiten por el financiamiento de la inversión de las empresas.

En la Tabla 1 se muestra una versión simple de este modelo que tiene cuatro ecuaciones; para una versión con mayores detalles, véase Dancourt (2012).

La ecuación (1) corresponde a la curva IS, donde se determina el nivel de actividad económica $(Y)$, si están dadas la tasa de interés de los préstamos bancarios $(R)$ y la tasa de interés del mercado de bonos (i). La ecuación (2) corresponde a la curva LM, donde se determina la cantidad de dinero $(M)$ si el banco central fija la tasa de interés de los bonos (i) y la tasa de encaje $(\theta)$. La ecuación (3) corresponde a la curva de oferta agregada $(\mathrm{OA})$, donde el nivel de precios depende directamente de la brecha del producto $(Y-\bar{Y})$ y de un choque de oferta $\left(P_{0}\right)$. Por último, la ecuación (4) corresponde a la curva LL, que representa el equilibrio en el mercado de préstamos bancarios, donde se determina la tasa de interés de los préstamos bancarios $(R)$, dados la actividad económica y el nivel de precios. Por la ley de Walras, no se considera el mercado de bonos.

Tabla 1

$$
\begin{aligned}
& Y=k\left[I_{0}+G-b(R+i)\right] \\
& M=\theta\left(P+Y-\alpha_{1} i\right) \\
& P=\alpha_{3} P_{0}+\alpha_{4}(Y-\bar{Y}) \\
& \lambda(1-\theta)\left(P+Y-\alpha_{1} i\right)=P+Y-\alpha_{2}(R-i)
\end{aligned}
$$

Conviene empezar la descripción de este modelo Bernanke-Blinder (B-B) por la ecuación (4), la curva LL, que es su componente peculiar.

En este modelo, $\left(P+Y-\alpha_{1} i\right)$ son los depósitos bancarios que no rinden interés; no hay circulante. El público divide su riqueza financiera entre depósitos bancarios, asociados a una tarjeta de débito, y bonos. La demanda de depósitos bancarios depende directamente del nivel de precios $(P)$ y la actividad económica $(Y)$ e, inversamente, de la tasa de interés de los bonos $(i)$, que es fijada por el banco central. Los fondos prestables, los depósitos menos los encajes, son $(1-\theta)\left(P+Y-\alpha_{1} i\right)$, donde $(\theta)$ es la tasa de encaje fijada por el banco central. Con estos fondos prestables, los bancos adquieren bonos del gobierno o extienden préstamos a las empresas, según cual sea la propensión a prestar

\footnotetext{
7 Si el sistema bancario y el mercado de bonos compiten por el ahorro de las familias, estos depósitos bancarios deberían pagar una tasa de interés positiva vinculada a la tasa de interés del mercado de bonos; sobre este tema, véase Stiglitz y Greenwald (2003), Cap. 4. Aquí se asume que esto no ocurre y que la tasa bancaria de interés pasiva es cero.
} 
de los banqueros $(\lambda)$, que está entre cero y uno ${ }^{8}$. En consecuencia, la oferta de préstamos bancarios en términos de stock $(L)$ está dada por $L=\lambda(1-\theta)\left(P+Y-\alpha_{1} i\right)$.

La demanda de préstamos bancarios en términos de stock $\left(L^{d}\right)$ está dada por $L^{d}=P$ $+Y-\alpha_{2}(R-i)$, donde $(R)$ es la tasa de interés de los préstamos bancarios, y donde se supone que las firmas tienen una fuente alternativa de crédito en el mercado de bonos al costo $(i)$, de tal modo que la demanda de préstamos bancarios se eleva cuando sube $(i)$. La demanda de préstamos también depende directamente del nivel de precios $(P)$ y de la actividad económica $(Y)$. La curva LL implica que la oferta de préstamos $(L)$ es igual a la demanda de préstamos $\left(L^{d}\right)$.

Respecto a la curva IS, si la inversión privada $(I)$ depende inversamente de estas dos tasas de interés que rigen en el mercado de préstamos bancarios $(R)$ y en el mercado de bonos $(i)$, podemos tener una función de inversión similar a la del libro de texto de macroeconomía dada por $I=I_{0}-b_{1} R-b_{2} i$, donde $I_{0}$ es la inversión autónoma. Se asume que la inflación esperada es nula. Esta función de inversión sería $I=I_{0}-b(R+i)$, si $b_{1}=b_{2}=b$. La función consumo está dada por $C=c(1-t) Y$, siendo $(1-t) Y$ el ingreso disponible y $(c)$ la propensión a consumir. El gasto público $(G)$ y la tasa del impuesto a la renta $(t)$ son los instrumentos de la política fiscal y el gobierno financia su déficit colocando bonos. Igualando la producción $(Y)$ y la demanda agregada $(C+I+G)$, obtenemos la ecuación (1), la curva IS, donde $k=1 /[1-c(1-t)]$ es el multiplicador keynesiano.

El nivel de precios $(P)$ está dado por la curva de oferta agregada $(\mathrm{OA})$ donde $(Y-\bar{Y})$ es la brecha entre el producto efectivo y el producto potencial. El nivel de precios sube en los auges y baja en las recesiones porque el mark-up y/o los salarios nominales son procíclicos. El término $\left(P_{0}\right)$ sirve para representar un choque de oferta como un cambio en el precio del petróleo.

La curva LM determina la cantidad de dinero $(M)$ si el banco central fija la tasa de interés $(i)$ de los bonos. Aquí, la LM surge de igualar la oferta de base monetaria $(M)$ con la demanda de base monetaria, (que es una demanda de encajes o reservas bancarias), dada por $\theta\left(P+Y-\alpha_{1} i\right)$, siendo $(\theta)$ la fracción de los depósitos bancarios $\left(P+Y-\alpha_{1} i\right)$ que los bancos comerciales están obligados a mantener ociosa en el banco central.

En la figura 1, se representa el equilibrio parcial en el mercado de préstamos bancarios, suponiendo que el nivel de precios $(P)$ y la actividad económica $(Y)$ están dados. Un alza de la tasa de referencia (i) traslada la oferta de préstamos hacia la izquierda $\left(L^{s 1}\right)$, porque reduce los depósitos y los fondos prestables; y, además, traslada la demanda de préstamos hacia la derecha $\left(L^{d 1}\right)$, porque encarece el financiamiento alternativo en el mercado de bonos; de este modo, se reduce el volumen de préstamos y se eleva la tasa de interés bancaria $(R)$. Vamos del punto A al punto B. Un alza de la tasa de encaje $(\theta)$

8 En el modelo B-B original, la propensión a prestar depende de la tasa de interés de los bonos y de los préstamos bancarios. Sin embargo, Bernanke y Blinder (1988) subrayan que «los choques a la oferta de crédito son comunes en la historia» $y$, en particular, que esta propensión a prestar tiende a desplomarse con las crisis financieras. 
en la magnitud adecuada solo traslada la oferta de préstamos hacia la izquierda, lo cual reduce el volumen de préstamos y eleva la tasa de interés bancaria $(R)$, aunque menos que en el caso anterior. Vamos del punto A al punto C. La tasa de referencia afecta el costo de endeudarse en el sistema bancario y en el mercado de bonos mientras que la tasa de encaje solo afecta el costo de endeudarse en el sistema bancario.

Figura 1. Alzas de la tasa de encaje y de la tasa de referencia

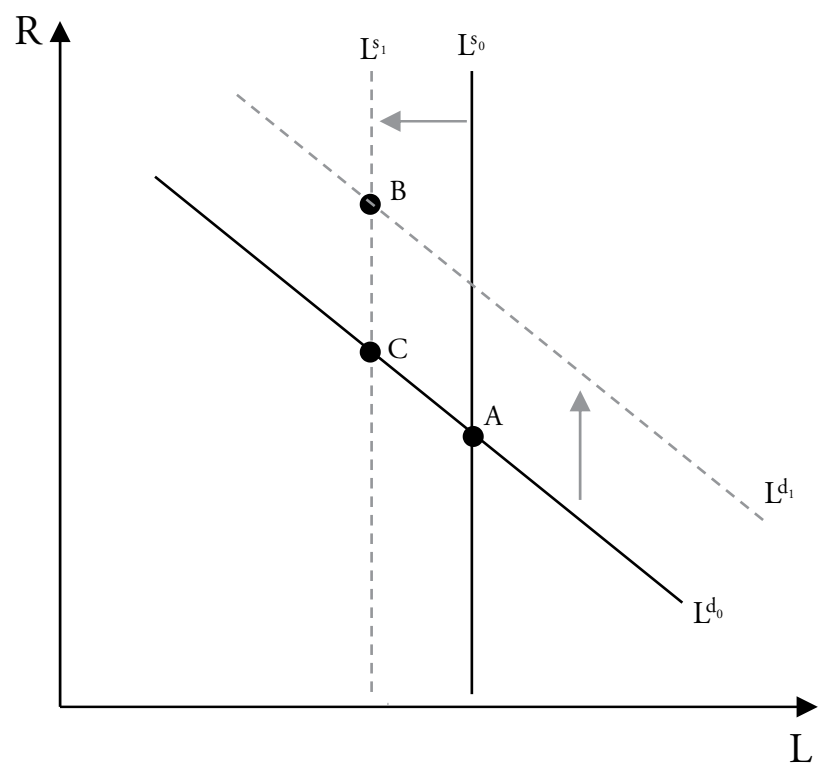

Si igualamos la oferta y la demanda de préstamos, insertando allí la ecuación de la oferta agregada $(\mathrm{OA})$, se obtiene la curva LL de la figura 2. Esta curva LL representa el equilibrio en el mercado de préstamos para distintas combinaciones de actividad económica $(Y)$ y tasa de interés bancaria $(R)$. La curva LL tiene pendiente positiva porque un incremento de la actividad económica $(Y)$ genera un exceso de demanda de préstamos que eleva la tasa de interés $(R)$ bancaria.

La ecuación de la curva LL es

$$
R=\left(1+\frac{\alpha_{0} \alpha_{1}}{\alpha_{2}}\right) i+\left(1+\alpha_{4}\right)\left(1-\alpha_{0}\right) \frac{1}{\alpha_{2}} Y
$$

Donde $0<\alpha_{0}<1$ siendo $\alpha_{0}=\lambda(1-\theta)$; y donde se ha supuesto que $\alpha_{3} P_{0}=\alpha_{4} \bar{Y}$, para simplificar la notación.

Un alza de la tasa de encaje $(\theta)$ o un alza de la tasa de referencia $(i)$ trasladan la curva LL en la figura 2 hacia la izquierda (línea punteada), es decir, elevan la tasa de interés bancaria $(R)$ para cualquier nivel de actividad económica $(Y)$, porque reducen la oferta de préstamos; el alza de la tasa de referencia, además, aumenta la demanda de préstamos. 
Figura 2. Alzas de la tasa de encaje y de la tasa de referencia

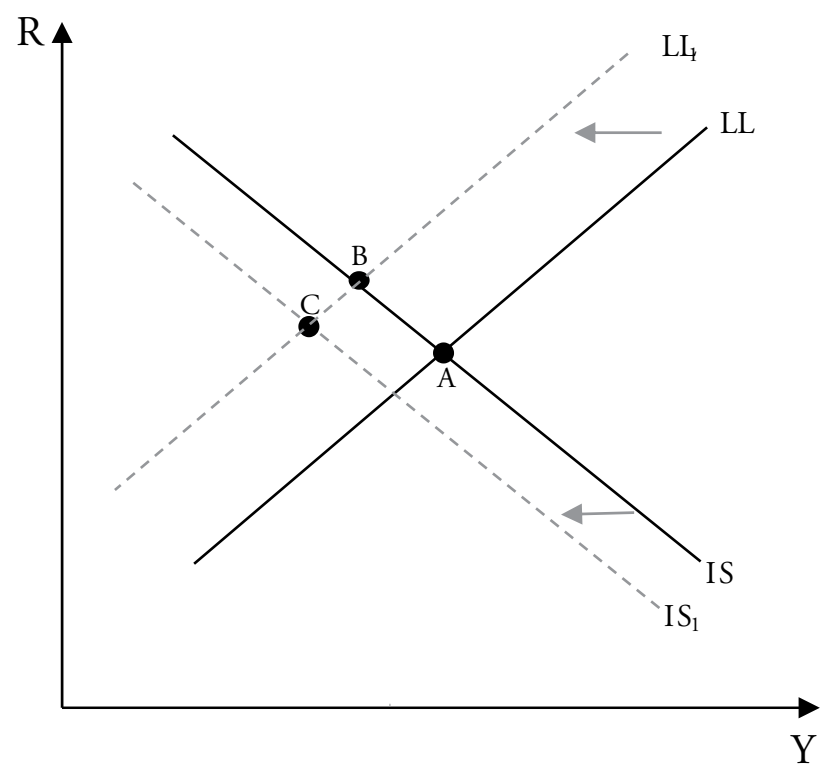

La curva IS tiene pendiente negativa en la figura 2 porque un alza de la tasa de interés bancaria $(R)$ reduce la inversión privada y, dado el multiplicador keynesiano, también la actividad económica $(Y)$. La curva IS se traslada a la izquierda (línea punteada) si sube la tasa de referencia ( $i$ ), porque eleva el costo de endeudarse en el mercado de bonos y reduce así la inversión privada y la actividad económica para cualquier tasa $(R)$ de interés bancaria; la IS no depende de la tasa de encaje.

Un alza de la tasa de referencia ( $i$ ) traslada las curvas IS y LL hacia la izquierda, en la figura 2. La actividad económica $(Y)$ cae y, dada la curva OA, el nivel de precios se reduce. Vamos del punto A al punto $C$. Hemos supuesto que la tasa de interés bancaria $(R)$ sube. Para que esto ocurra basta que la demanda de préstamos sea suficientemente sensible al spread entre $R$ e $i$; es decir, $\alpha_{2}=\left(1+\alpha_{4}\right) k b$. Este parámetro $\alpha_{2}$ mide, digamos, el grado de competencia existente entre el sistema bancario y el mercado de bonos.

Un alza de la tasa de encaje $(\theta)$ de la magnitud adecuada solo traslada la curva LL hacia la izquierda, en la figura 2 , de tal manera que cae el nivel de actividad económica (menos que con el alza de la tasa de referencia) y sube la tasa de interés bancaria (más que con el alza de la tasa de referencia). Vamos del punto A al punto B. Dada la curva OA, el nivel de precios también se reduce.

En suma, una política monetaria restrictiva (expansiva), implementada vía la tasa de interés de referencia o vía la tasa de encaje, produce cualitativamente los mismos resultados: frena (impulsa) la actividad económica, reduce (eleva) el nivel de precios, y aumenta (disminuye) la tasa activa de interés bancaria. 
Este modelo Bernanke-Blinder permite derivar el impacto total sobre la actividad económica y la tasa activa de interés bancaria de un cambio en la tasa de referencia o en la tasa de encaje, ceteris paribus. Los multiplicadores que vinculan el cambio en ambos instrumentos de la política monetaria $(d i, d \theta)$ con el cambio en la actividad económica y en la tasa activa de interés bancaria $(d Y, d R)$, se presentan a continuación?

$$
\begin{aligned}
& \frac{d Y}{d i}=\frac{-\left(\alpha_{0} \alpha_{1}+2 \alpha_{2}\right)}{\frac{\alpha_{2}}{k b}+\left(1-\alpha_{0}\right)\left(1+\alpha_{4}\right)}<0 \\
& \frac{d Y}{d \theta}=\frac{-\left(1+\alpha_{4}\right) \lambda Y}{\frac{\alpha_{2}}{k b}+\left(1-\alpha_{0}\right)\left(1+\alpha_{4}\right)}<0 \\
& \frac{d R}{d i}=\frac{\frac{\alpha_{0}}{k b}\left(\alpha_{1}+\alpha_{2}\right)}{\frac{\alpha_{2}}{k b}+\left(1-\alpha_{0}\right)\left(1+\alpha_{4}\right)}>0 \\
& \frac{d R}{d \theta}=\frac{\frac{\left(1+\alpha_{4}\right) \lambda Y}{k b}}{\frac{\alpha_{2}}{k b}+\left(1-\alpha_{0}\right)\left(1+\alpha_{4}\right)}>0
\end{aligned}
$$

La eficacia relativa de ambos instrumentos de la política monetaria, en términos de sus efectos sobre la actividad económica, depende de la estructura financiera. Igualando el primer y el segundo resultado, se obtiene que

$$
d \theta=\frac{\left(\alpha_{0} \alpha_{1}+2 \alpha_{2}\right)}{\left(1+\alpha_{4}\right) \lambda Y} d i
$$

Mientras mayor sea la competencia entre el sistema bancario y el mercado de bonos, es decir, mientras mayor sea $\alpha_{2}$, todo lo demás constante, menor será la eficacia relativa de la tasa de encaje $(d \theta)$ respecto de la tasa de referencia $(d i)$ para obtener un mismo cambio en el nivel de actividad económica.

Finalmente, conviene especificar en qué condiciones un alza de la tasa de encaje $(\theta)$ no tiene efecto alguno sobre la tasa activa de interés bancaria. Supongamos que el banco central eleva la tasa de encaje $(\theta)$ para reducir la oferta de crédito $(L)$, contra la voluntad de los banqueros, mientras mantiene constante la tasa de interés de referencia $(i)$ o tasa de política. Si los banqueros no quieren reducir sus préstamos, a pesar de la disminución de los fondos prestables generada por el alza de la tasa de encaje, eso implica que

\footnotetext{
9 Para hallar la derivada total $d R / d i$ asumimos que $\alpha_{2}=\left(1+\alpha_{4}\right) k b$. También se pueden hallar los multiplicadores que vinculan el cambio en ambos instrumentos de la política monetaria $(d i, d \theta)$ con los cambios en la base monetaria $(d M)$, en el nivel de precios $(d P)$ o en la oferta de crédito bancario $(d L)$.
} 
su propensión a prestar $(\lambda)$ debe aumentar. $\mathrm{Si}(\lambda)$ aumenta en la magnitud apropiada, dada la tasa de interés $(i)$ de los bonos, el alza de la tasa de encaje $(\theta)$ solo conduce a un aumento de la base monetaria y de los depósitos bancarios, sin afectar la tasa activa de interés bancaria, la actividad económica o la oferta de préstamos.

La condición para que ocurra lo anterior es que $\alpha_{0}$ no varíe, siendo $\alpha_{0}=\lambda(1-\theta)$. En este caso, las curvas IS y LL no se mueven en la figura 2. Esto significa que los bancos comerciales venden sus bonos al banco central, para poder mantener constante el volumen de préstamos, cuando este sube la tasa de encaje y reduce los fondos prestables. En este caso, el banco central se ve obligado a comprar estos bonos, suministrándoles así a los bancos comerciales los fondos prestables requeridos, si quiere mantener fija la tasa de interés de política $(i)$.

\section{EL PROCEDIMIENTO ECONOMÉTRICO}

En el Perú, la evaluación empírica del impacto de los instrumentos de política monetaria (tasa de referencia y tasa de encaje) sobre las tasas de interés activas y pasivas del sistema bancario, no ha utilizado datos de bancos individuales; solamente se ha enfocado en un instrumento de política (la tasa de referencia) y en el comportamiento de la tasa promedio de interés activa (distinguiendo por plazos y tipo de crédito) del sistema bancario. Trabajos importantes al respecto son el de Lahura (2006), el del Reporte de Inflación de junio de 2009 (BCRP, 2009), y el de Armas, Castillo y Vega (2014) que evalúa ambos instrumentos de política monetaria.

Los trabajos que han utilizado datos de bancos individuales son escasos, aún a nivel internacional. Heffernan (1997), es uno de los pioneros que estudia, con un modelo de corrección de errores, la velocidad de ajuste de las distintas tasas activas y pasivas fijadas por los bancos comerciales y otras entidades financieras inglesas ante un cambio en la tasa de interés de referencia o tasa de política del Banco de Inglaterra.

Los tres trabajos más importantes que utilizan un modelo de panel dinámico para determinar el impacto de la tasa de referencia sobre las tasas de interés cobradas por distintos bancos, son los de Weth (2002) para Alemania, Gambacorta $(2004)^{10}$ para Italia y Bernstein y Fuentes (2003) para Chile. También se puede mencionar el trabajo de Vaugnet, Collin y Dhynne (2007) para Bélgica. Nuestro modelo empírico está basado en el trabajo de Weth (2002).

El modelo dinámico de datos de panel permite usar la información microeconómica individual para caracterizar la variable endógena: la tasa de interés activa de cada entidad bancaria. La idea básica es que la tasa activa de interés bancaria depende directamente de la tasa de interés de referencia y de la tasa de encaje en $\mathrm{MN}$ en el equilibrio estacionario,

${ }^{10}$ Gambacorta (2004) incluye características individuales de los bancos (tamaño, morosidad, liquidez) como variables de control del modelo. Estimamos para el caso peruano un modelo similar al planteado por Gambacorta (2004), pero estas características individuales no resultaron relevantes en la estimación. 
como en el modelo Bernanke-Blinder visto en la sección anterior. Si, por ejemplo, sube la tasa de referencia, eso provocará que también suban las tasas activas de interés de los distintos bancos y se espera, siguiendo a la literatura, que el coeficiente de traspaso sea igual a uno en el largo plazo. En el tránsito de un equilibrio estacionario a otro, se espera que las tasas de interés activas muestren un cierto grado de inercia y se ajusten con mayor o menor lentitud a los cambios en los instrumentos de la política monetaria ${ }^{11}$.

Si prescindimos de las variables de control, la idea que captura la ecuación usada por Weth (2002) es que la tasa de interés activa $\left(r_{i t}\right)$ del banco i en el periodo t depende de sus propios rezagos, y del instrumento de política monetaria $\left(m_{t-j}\right)$, actual y rezagado, más un término de error y una constante $\left(c_{i}\right)$ propia de cada banco. Es decir,

$$
r_{i t}=c_{i}+\sum_{j=1}^{k} \alpha_{j} r_{i t-j}+\sum_{j=0}^{k} \beta_{j} m_{t-j}+\epsilon_{i t}
$$

Siguiendo a Weth (2002), existe un equilibrio estacionario, para k=3, si:

$$
\begin{aligned}
& \alpha_{1}+\alpha_{2}+\alpha_{3}<1 \\
& \beta_{0}+\beta_{1}+\beta_{2}+\beta_{3}>0
\end{aligned}
$$

La ecuación (6) se puede expresar alternativamente como un modelo de corrección de errores; es decir,

$$
\Delta r_{i t}=\mu_{i}+\sum_{j=1}^{3} \varphi_{j} \Delta r_{i t-j}+\sum_{j=0}^{3} \omega_{j} \Delta m_{t-j}+(\delta+\gamma)\left[r_{i t-1}+\frac{\gamma}{\delta+\gamma}\left(r_{i t-1}-m_{t-1}\right)\right]+\varepsilon_{i t}(7)
$$

Donde: $\quad \varphi_{1}=-\left(\alpha_{2}+\alpha_{3}\right) ; \varphi_{2}=-\alpha_{3}$

$$
\begin{aligned}
& \omega_{0}=\beta_{0} ; \omega_{1}=-\left(\beta_{2}+\beta_{3}\right) ; \omega_{2}=-\beta_{3} \\
& \gamma=-\left(\beta_{0}+\beta_{1}+\beta_{2}+\beta_{3}\right) \\
& \delta=-\left(1-\alpha_{1}-\alpha_{2}-\alpha_{3}\right)+\left(\beta_{0}+\beta_{1}+\beta_{2}+\beta_{3}\right)
\end{aligned}
$$

Como señala Weth (2002), la relación en el equilibrio estacionario, o de largo plazo, entre la tasa activa y la tasa de referencia está dada por $\frac{\gamma}{\delta+\gamma}$. El denominador de esta fracción debe ser negativo si la tasa activa tiende al equilibrio estacionario después de una modificación de los instrumentos de política monetaria. Los cambios en las tasas activas de los distintos bancos difieren entre sí de acuerdo al intercepto $\mu_{i}$, cada uno de los cuales se consideran como efectos fijos individuales.

La estimación del panel dinámico se realiza mediante el estimador clásico, de mínimos cuadrados con variables dummy (conocido como estimador LSDV, por sus siglas en inglés), las cuales justamente permiten modelar la heterogeneidad individual paramétricamente. Es importante notar que, teóricamente, la inclusión de la variable endógena

11 Véase al respecto, la discusión en Lahura (2006), Bernstein y Fuentes (2003) y Weth (2002). 
rezagada hace que el estimador LSDV sea sesgado cuando la dimensión temporal del panel es pequeña. Sin embargo, Judson y Owen (1999) muestran que el sesgo se diluye para periodos de tiempo mayores a 30. Asimismo, este estimador presenta mejores propiedades asintóticas cuando el número de individuos en la muestra es relativamente pequeño y el número de periodos de tiempo es relativamente grande; en el caso contrario, el estimador GMM es superior ${ }^{12}$. En nuestro caso, los periodos de tiempo son 81 (junio 2003-junio 2010) y el número de individuos es 6, lo cual permite usar el estimador LSDV de manera confiable. Por otra parte, en la estimación se consideran varios rezagos de las variables explicativas, lo cual permite controlar razonablemente por potenciales problemas de endogeneidad.

Los datos utilizados en la estimación econométrica son de frecuencia mensual y corresponden al periodo junio 2003-junio 2010. Las variables financieras se obtienen de la Superintendencia de Banca y Seguros (SBS) y las variables macroeconómicas del Banco Central de Reserva del Perú (BCRP).

La base de datos proporcionada por la SBS cuenta con información diaria de las tasas de interés activas, efectiva anual promedio, y de los flujos desembolsados en $\mathrm{MN}$ y $\mathrm{ME}$, que ha sido remitida por cada institución financiera, de acuerdo a los formatos del reporte 6-D y 6E, vigentes para el periodo comprendido entre setiembre de 2002 julio de 2012. Sobre la base de esta información se construyen ${ }^{13}$ las tasas de interés activas promedio para distintos plazos de los préstamos comerciales. Se consideran los siguientes plazos: hasta 360 días, hasta 180 días, hasta 90 días y hasta 30 días.

Para la estimación econométrica básica se utiliza la tasa de interés en $\mathrm{MN}$ para créditos comerciales hasta 360 días. Los créditos comerciales representan el $72 \%$ del total del crédito otorgado en $\mathrm{MN}$ por el sistema bancario al sector privado ${ }^{14}$. La muestra seleccionada es un panel balanceado de los seis bancos más grandes del país, que explican el $94 \%$ del crédito comercial en $\mathrm{MN}$ del sistema bancario al sector privado ${ }^{15}$, y abarca el periodo comprendido entre junio 2003-junio 2010. Como sugiere Gambacorta (2004), los resultados que se obtengan en este caso para el banco promedio tendrán más sentido macroeconómico, que en el caso alternativo donde se incluyeran en la muestra también los bancos más pequeños y las cajas municipales. La idea es medir el impacto que la política monetaria tiene sobre las tasas de interés activas en moneda nacional fijadas por los bancos grandes, que otorgan una fracción sustancial del crédito del sistema financiero.

\footnotetext{
12 El método GMM, propuesto por Arellano y Bond (1991), permite controlar los efectos no observados por banco y la potencial endogeneidad de las variables explicativas.

${ }^{13}$ A partir de las tasas de interés diarias y flujos desembolsados, se calcula el interés generado por monto desembolsado. Se hace la agregación mensual, para luego calcular la tasa de interés efectiva anualizada según plazo del préstamo.

${ }_{14}$ Se consideran préstamos y descuentos. El crédito total se divide en 4 categorías: comercial, microempresa, consumo e hipotecario.

${ }^{15}$ Los bancos incluidos son: Banco Interamericano de Finanzas, Citibank, Banco Continental, Banco de Crédito, Interbank y Scotiabank.
} 
En el Gráfico 1 se muestra la dispersión de las tasas de interés para préstamos hasta 360 días, por institución financiera. Mientras que en el Gráfico 2 se muestra la evolución temporal de las tasas de interés de los créditos comerciales de estos seis bancos y su relación con la tasa de interés de referencia del $\mathrm{BCRP}^{16}$.

Gráfico 1. Tasa de interés de créditos comerciales (2003-2010)

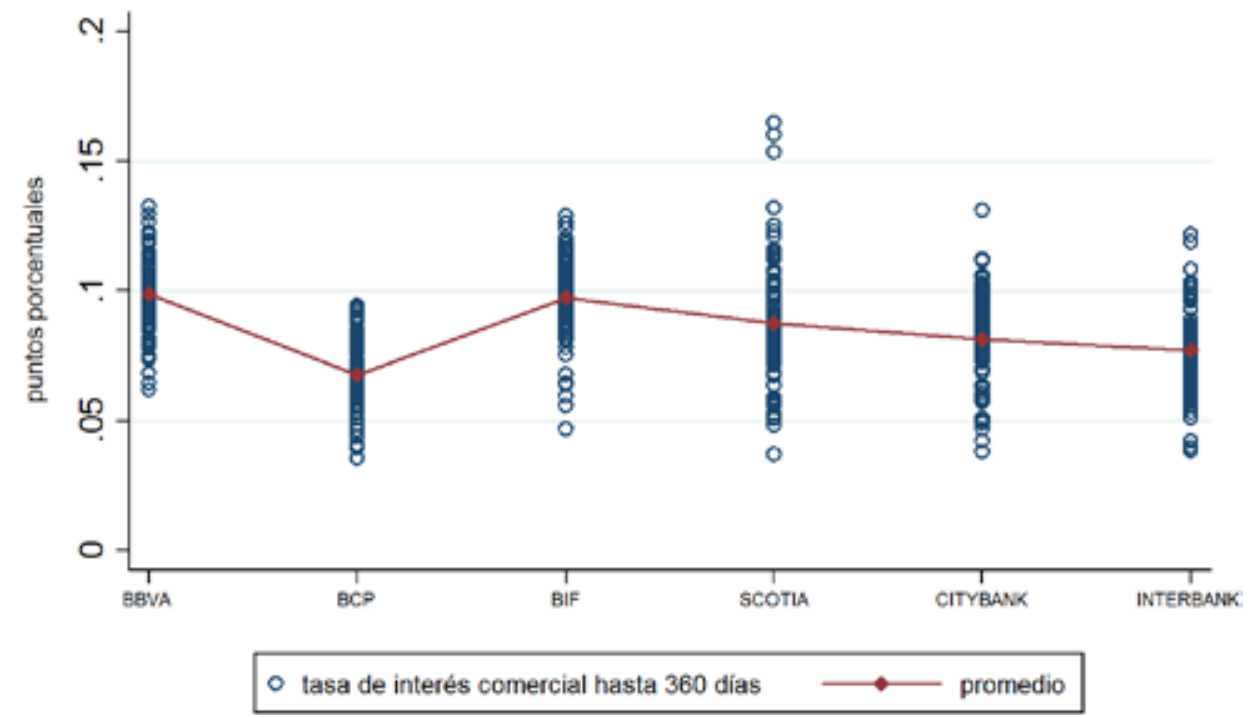

Fuente: SBS. Elaboración propia.

Gráfico 2. Tasa de interés de créditos comerciales y tasa de referencia (2003-2010)
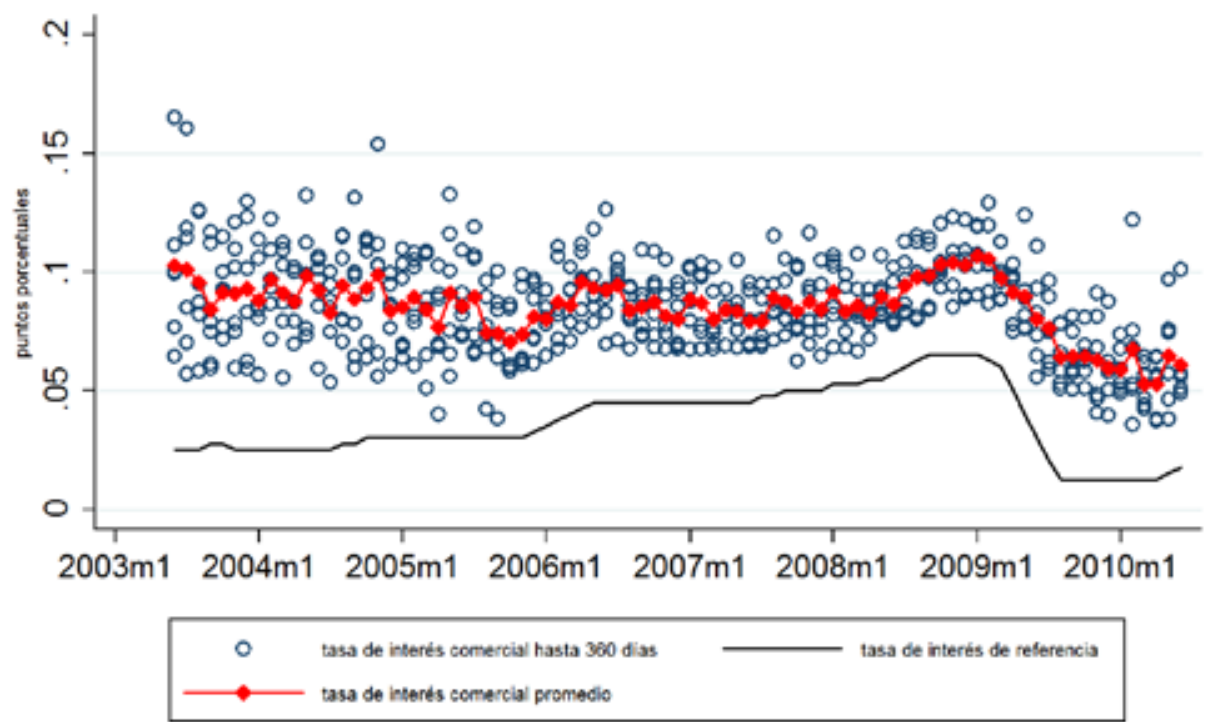

Fuente: SBS. Elaboración propia.

16 En el anexo 2 se presentan los gráficos para las tasas de interés considerando distintos plazos. 
Finalmente, cabe mencionar que se aplicaron los test de raíz unitaria a las tasas de interés activas en $\mathrm{MN}$ y ME, la tasa de interés interbancaria, y a la tasa de encaje en moneda nacional, todos en niveles. Así mismo, debido a que es altamente probable que exista correlación cruzada entre las tasas de interés de los distintos bancos, dado que tienen un comportamiento muy similar, y que ello pueda distorsionar los resultados de las pruebas, también se consideran las desviaciones de las tasas de interés de cada banco con respecto al promedio de las tasas de todos los bancos en cada periodo (promedio de sección cruzada). En el Anexo 2 se presenta ambos juegos de resultados. En el caso de las tasas de interés activas así como la tasa de interés en dólares, la evidencia sugiere que estas son variables estacionarias tanto en niveles como en sus desviaciones respecto al promedio de sección cruzada. En el caso de la tasa de interés interbancaria si bien la evidencia para sus niveles es mixta, sus desviaciones respecto al promedio de sección cruzada muestran un comportamiento consistente con estacionariedad. En el caso de la tasa de encaje, los resultados sugieren que esta serie presenta raíz unitaria. Sin embargo, después de analizar detalladamente el comportamiento de esta variable, consideramos que este resultado podría deberse a los pocos movimientos de la tasa de encaje durante los años 2003-2008 y a los fuertes cambios ocurridos a partir de esa fecha. Por esta razón, y teniendo en cuenta la naturaleza acotada de esta variable, en lo que sigue asumiremos que esta variable es estacionaria.

\section{RESULTADOS}

En esta sección se detallan los resultados de la estimación del modelo de datos de panel utilizando las tasas de interés en moneda nacional $(\mathrm{MN})$ de los créditos comerciales para distintos plazos.

Como primera aproximación, se realizó la estimación del modelo de datos de panel descrito en la ecuación (6), utilizando las tasas de interés en MN para créditos comerciales hasta 360 días, y los dos instrumentos de política monetaria (la tasa de encaje en MN y la tasa de interés interbancaria en MN). También se utilizó la tasa de interés en moneda extranjera (ME) para créditos comerciales hasta 360 días, dada la dolarización del crédito del sistema bancario (véase Dancourt, 2014). El modelo incluye tres rezagos tal como se especifica a continuación.

$$
r_{i t}=c_{i}+\sum_{j=1}^{3} \alpha_{j} r_{i t-j}+\sum_{j=0}^{3} \beta_{j} i_{t-j}+\sum_{j=0}^{3} \beta_{j}^{\theta} \theta_{t-j}+\sum_{j=0}^{3} \beta_{j}^{\$} i_{t-j}^{\$}+\epsilon_{i t}
$$

Donde $r_{i t}$ es la tasa de interés activa, $i_{t-j}$ es la tasa de interés interbancaria en $\mathrm{MN}, \theta_{t}$ es la tasa de encaje en $\mathrm{MN}$, e $i_{t}^{\$}$ es la tasa de interes activa en ME.

Los resultados de la estimación por LSDV se muestran en la Tabla 2, siendo destacables algunos hallazgos. En primer lugar, el impacto de la tasa de interés interbancaria 
sobre la tasa de interés activa en MN hasta 360 días fijada por el banco promedio es positivo y estadísticamente significativo.

Tabla 2. Efectos sobre la tasa de interés de créditos comerciales en MN hasta 360 días

\begin{tabular}{lll}
\hline & Coef & Error estàndar \\
\hline
\end{tabular}

Tasa de interés comercial en MN hasta 360 días

$$
\sum_{j=1}^{3} \alpha_{j} \quad 0,713709^{* * *} \quad 0,0428149
$$

Tasa de interés interbancaria en MN

$$
\sum_{j=0}^{3} \beta_{j}
$$

Tasa de encaja en MN

$$
\sum_{j=0}^{3} \beta_{j}^{\theta}
$$

0,0201901

Tasa de interés comercial en ME hasta 360 días

$$
\sum_{j=0}^{3} \beta_{j}^{\$}
$$

Resultados del modelo panel dinámico con efectos fijos, estimado por LSDV.

Número de observaciones $=492$. Número de grupos $=6$.

Significativo al $10 \%(*), 5(*), 1 \%(* *)$.

Modelo: $r_{i t}=c_{i}+\sum_{j=1}^{3} \alpha_{j} r_{i t-j}+\sum_{j=0}^{3} \beta_{j} i_{i t-j}+\sum_{j=0}^{3} \beta_{j}^{\theta} \theta_{t-j}+\sum_{j=0}^{3} \beta_{j}^{\$} i_{t-j}^{\$}+\epsilon_{i t}$

En segundo lugar, no se encuentra evidencia que sugiera que la tasa de encaje en MN afecta la tasa de interés activa en MN hasta 360 días. En tercer lugar, la tasa de interés activa en moneda extranjera del mismo plazo tiene un impacto positivo, aunque no es estadísticamente significativo, sobre la tasa de interés activa en MN hasta 360 días.

Otro aspecto destacable de los resultados mostrados en la Tabla 2 es que los coeficientes de la variable dependiente rezagada son positivos y estadísticamente significativos en promedio (aunque no son reportados, cada uno de ellos también es positivo y estadísticamente significativo), permitiendo caracterizar a la dinámica de las tasas de interés activas cobradas por los bancos peruanos como un proceso altamente persistente pero estable, lo cual concuerda con lo que se ha observado en otros países. 
Tabla 3. Efectos sobre la tasa de interés de créditos comerciales en MN hasta 360 días

\begin{tabular}{lll}
\hline & Coef & Error estàndar \\
\hline
\end{tabular}

Tasa de interés comercial en MN hasta 360 días

$$
\sum_{j=1}^{3} \alpha_{j} \quad 0,713796^{* * *} \quad 0,0424388
$$

Tasa de interés interbancaria en MN

$$
\sum_{j=0}^{3} \beta_{j} \quad 0,184926^{* * *} \quad 0,0425989
$$

Tasa de interés comercial en ME hasta 360 días

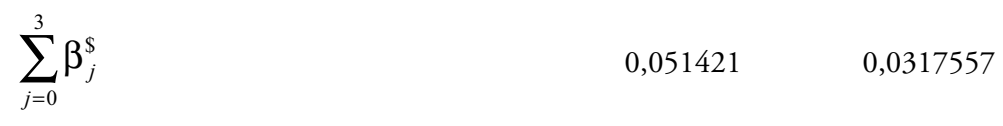

Resultados del modelo panel dinámico con efectos fijos, estimado por LSDV.

Número de observaciones $=492$. Número de grupos $=6$.

Significativo al $10 \%(*), 5(*), 1 \%\left({ }^{* *}\right)$.

Modelo: $r_{i t}=c_{i}+\sum_{j=1}^{3} \alpha_{j} r_{i t-j}+\sum_{j=0}^{3} \beta_{j} i_{i t-j}+\sum_{j=0}^{3} \beta_{j}^{\$} i_{t-j}^{\$}+\epsilon_{i t}$

La Tabla 3 muestra los resultados de la estimación excluyendo la tasa de encaje en MN de la ecuación (3). Como se puede observar, el impacto de la tasa de interés activa en ME hasta 360 días sobre la tasa de interés activa en MN del mismo plazo, es positivo y significativo, resultado que concuerda con el hecho de que los préstamos en MN y ME son sustitutos entre sí. Como también se puede observar en la Tabla 3, los coeficientes estimados de la variable dependiente rezagada y de la tasa de interés interbancaria en MN son similares a los reportados en la Tabla 1, lo cual da robustez a los resultados comentados anteriormente.

Finalmente, centramos el análisis en la relación entre la tasa de interés interbancaria y la tasa de interés de los créditos comerciales en $\mathrm{MN}$ para distintos plazos ( 0 a 30 días, 0 a 90 días, 0 a 180 días y 0 a 360 días), excluyendo el resto de variables. Considerando las ecuaciones (6) y (7), se calculan las elasticidades de corto y largo plazo, así como la velocidad de ajuste de las tasas de interés activas ante desequilibrios en la relación de largo plazo. 
Tabla 4. Efectos sobre la tasa de interés de créditos comerciales en $\mathrm{MN}$ para distintos plazos

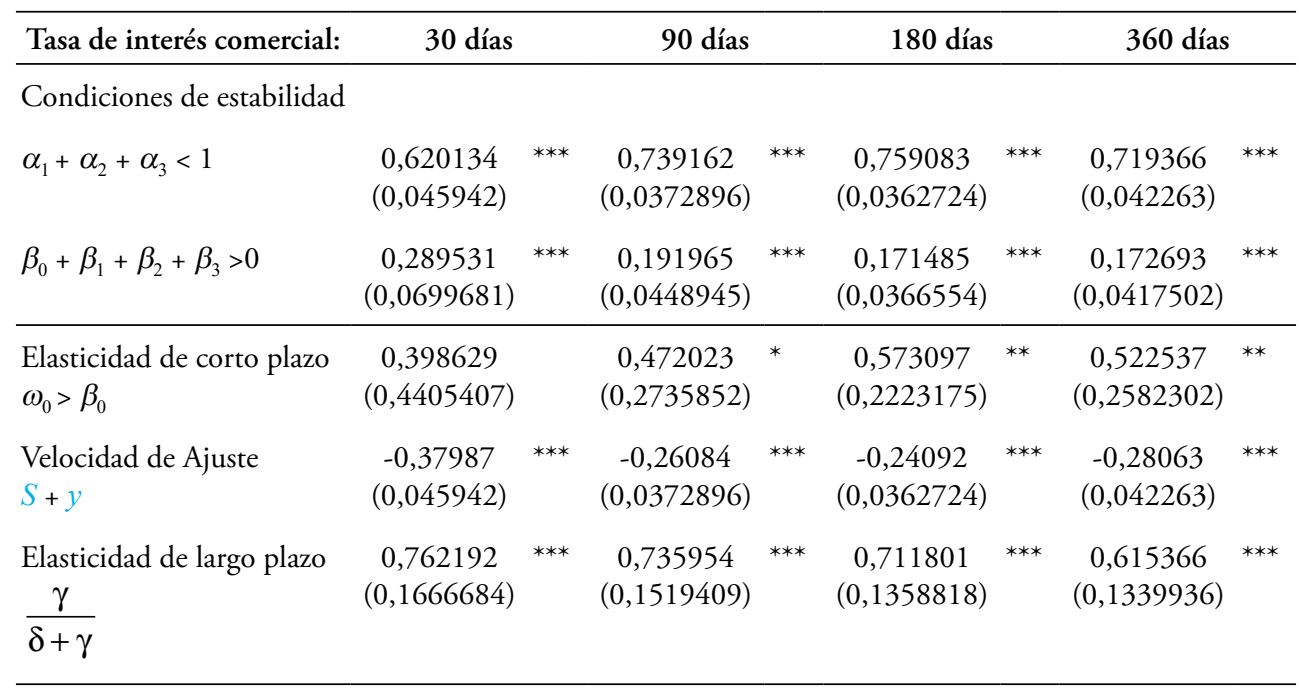

Resultados del modelo panel dinámico con efectos fijos, estimado por LSDV.

Número de observaciones $=492$. Número de grupos $=6$.

Error estándar en paréntesis.

Significativo al $10 \%\left({ }^{*}\right), 5 \%\left({ }^{*}\right), 1 \%\left({ }^{* *}\right)$.

Modelo:

$$
\begin{aligned}
& r_{i t}=c_{i}+\sum_{j=1}^{3} \alpha_{j} r_{i t-j}+\sum_{j=0}^{3} \beta_{j} m_{t-j}+\epsilon_{i t} \\
& \Delta r_{i t}=\mu_{i}+\sum_{j=1}^{3} \varphi_{j} \Delta r_{i t-j}+\sum_{j=0}^{3} \omega_{j} \Delta m_{t-j}+(\delta+\gamma)\left[r_{i t-j}+\frac{\gamma}{\delta+\gamma}\left(r_{i t-1}-m_{t-1}\right)+\epsilon_{i t}\right.
\end{aligned}
$$

Los resultados se reportan en la Tabla 4, donde se puede distinguir algunos aspectos interesantes. En primer lugar, salvo en el caso de los préstamos a mayor plazo ( 0 a 360 días vs. 0 a 180 días), el impacto de corto plazo de la tasa de referencia es mayor mientras mayor sea el plazo considerado. Este resultado no es común en la literatura. Por el contrario, para los préstamos de mayor plazo (360 días vs. 180 días), el impacto de la tasa de referencia sobre las tasas activas es menor mientras mayor sea el plazo de estas. Valdría la pena indagar con más detalle como los bancos grandes traspasan a sus tasas activas cambios en la tasa de interés de referencia, en el corto plazo.

Otro resultado destacable es que, salvo en el caso de los préstamos a mayor plazo, la velocidad de ajuste decrece a medida que aumenta el plazo de las tasas activas. (La excepción es la tasa de interés para préstamos de 0 a 360 días, cuya velocidad de ajuste es mayor a la de los préstamos de 0 a 180 días). 
Por último, tal como se espera, las elasticidades de largo plazo de las tasas activas con respecto a la tasa de referencia son mayores a las correspondientes elasticidades de corto plazo, excepto para los préstamos de 0 a 360 días. Estas elasticidades de largo plazo disminuyen mientras mayor es el plazo de los préstamos; y son menores que la unidad, lo cual indicaría que no es completo el ajuste de las tasas activas bancarias ante cambios en la tasa de interés de referencia.

\section{CONCLUSIONES}

El objetivo de este trabajo ha sido determinar el impacto de dos instrumentos de la política monetaria peruana sobre las tasas activas bancarias, estimando un modelo de panel dinámico con información mensual para los seis bancos más grandes del país, durante el periodo 2003-2010, caracterizado por un régimen monetario de metas de inflación.

Primero, este texto encuentra que un alza de la tasa de interés de referencia tiene un impacto positivo y estadísticamente significativo sobre las tasas de interés de los préstamos comerciales de 0 a 360 días fijadas por los seis bancos más grandes del país durante el periodo junio de 2003-junio de 2010.

Segundo, este texto no encuentra evidencia que sugiera que la tasa de encaje a los depósitos en $\mathrm{MN}$ influye de alguna manera sobre las tasas de interés de los préstamos comerciales de 0 a 360 días fijadas por los seis bancos más grandes del país durante el periodo junio de 2003-junio de 2010.

Estos resultados sugieren que la tasa de interés de referencia debería ser el principal instrumento de política monetaria del BCRP. 


\section{Anexo 1. Test de raíz unitaria}

Contraste de raíz unitaria: Intercepto (series en niveles)

\begin{tabular}{|c|c|c|c|c|c|c|c|c|c|c|c|c|c|c|}
\hline & $30 \mathrm{di}$ & & 90 días & & 180 días & & 360 días & & Interbanca & $\operatorname{aria} t$ & tasa dólare & & & \\
\hline Levin, Lin y Chu ${ }^{1 /}$ & $-3,677$ & & $-0,9974$ & & $-0,585$ & & $-3,6782$ & & $-3,307$ & $* *$ & $-6,742$ & $* *$ & 0,8597 & \\
\hline Breitung ${ }^{1 /}$ & $-3,9937$ & & $-2,7859$ & * & $-2,497$ & * & $-4,1516$ & $* *$ & $-0,7852$ & & $-7,2345 *$ & $* *$ & $-2,074$ & * \\
\hline Im, Pesaran y Shin ${ }^{2 /}$ & $-6,6182$ & ** & $-5,3693$ & $* *$ & $-5,243$ & $* *$ & $-6,3792$ & $* *$ & 3,432 & & $-6,3759 *$ & ** & 0,1253 & \\
\hline & 76,1866 & $* *$ & 57,4312 & $* *$ & 53,6649 & $* *$ & 56,7271 & $* *$ & 69,2144 & $* *$ & 87,9349 * & ** & 48,924 & ** \\
\hline $3 /$ & 3,8553 & ** & 3,8851 & $* *$ & 3,7177 & $* *$ & 3,7911 & ** & 0,0663 & & 1,9087 & * & 1,2638 & \\
\hline
\end{tabular}

${ }^{1 /}$ Ho: los miembros del panel contienen raíz unitaria. Ha: los miembros del panel son estacionarios.

${ }^{2 /}$ Ho: todos los miembros del panel contienen raíz unitaria. Ha: algunos miembros del panel son estacionarios.

${ }^{3 /}$ Ho: todos los miembros del panel son estacionarios. Ha: algunos miembros del panel contienen raíz unitaria.

${ }^{4 /}$ El estadístico P sigue una distribución Chi-cuadrado inversa.

* Se rechaza la Ho al $5 \%$. ${ }^{* *}$ Se rechaza la Ho al $1 \%$.

Contraste de raíz unitaria: Intercepto y tendencia (series en niveles)

\begin{tabular}{|c|c|c|c|c|c|c|c|c|c|c|c|c|c|}
\hline & 30 días & & 90 días & & 180 día & & 360 día & & Interbanca & & tasa dóla & & encaje \\
\hline Levin, Lin y Chu ${ }^{1 /}$ & $-6,2938$ & & $-0,5781$ & & 0,2249 & & $-2,5282$ & ** & $-2,7747$ & $* *$ & $-7,5677$ & ** & 0,9478 \\
\hline Breitung ${ }^{1 /}$ & $-4,0742$ & & $-3,4268$ & $* *$ & $-3,4949$ & ** & $-4,8048$ & ** & 5,4265 & & $-4,8864$ & ** & $-0,656$ \\
\hline Im, Pesaran y Shin ${ }^{2 /}$ & $-7,4558$ & $* *$ & $-6,3633$ & $* *$ & $-6,211$ & ** & $-7,61$ & ** & 4,1629 & & $-7,187$ & ** & $-0,557$ \\
\hline Fisher-ADF ${ }^{2 / 4 / 5 /}$ & 36,5771 & ** & 20,6783 & $*$ & 17,8567 & & 22,2717 & * & 16,4099 & & 45,5154 & ** & 11,692 \\
\hline Fisher-PP 2/ 4/ 5/ & 93,3307 & ** & 65,2451 & ** & 60,9693 & ** & 90,6859 & ** & 0,4312 & & 72,5883 & $* *$ & 5,6286 \\
\hline Hadri $^{3 /}$ & 2,7892 & ** & 2,9101 & . & 2,184 & * & 1,8607 & * & 4,1406 & ** & 0,2052 & & 2,0448 \\
\hline
\end{tabular}

${ }^{1 /}$ Ho: los miembros del panel contienen raiz unitaria. Ha: los miembros del panel son estacionarios.

${ }^{2 /}$ Ho: todos los miembros del panel contienen raiz unitaria. Ha: algunos miembros del panel son estacionarios.

${ }^{3 /}$ Ho: todos los miembros del panel son estacionarios. Ha: algunos miembros del panel contienen raíz unitaria.

${ }^{4 /}$ El estadístico P sigue una distribución Chi-cuadrado inversa.

${ }^{5 /}$ Solo tendencia.

* Se rechaza la Ho al 5\%. ${ }^{* *}$ Se rechaza la Ho al $1 \%$.

Debido a que las tasas de interés de los bancos analizados presentan un comportamiento similar, los resultados podrían estar afectados por la correlación cruzada entre las tasas de interés de los distintos bancos. Una solución a este problema es remover los promedios (promedios de sección cruzada) para controlar la posible correlación cruzada entre cada par de bancos. Los siguientes cuadros muestran el contraste de raíz unitaria, realizando el procedimiento descrito previamente. 


\section{Contraste de raíz unitaria: Intercepto (series en niveles)}

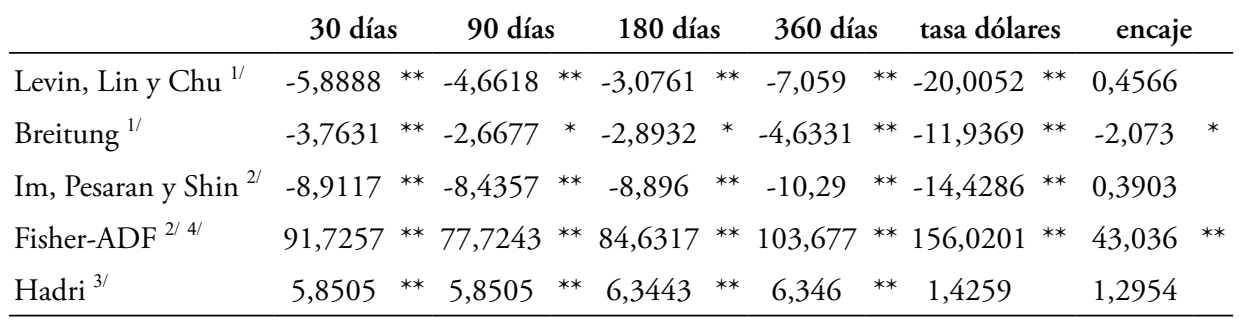

${ }^{1 /}$ Ho: los miembros del panel contienen raíz unitaria. Ha: los miembros del panel son estacionarios.

${ }^{2 /}$ Ho: todos los miembros del panel contienen raíz unitaria. Ha: algunos miembros del panel son estacionarios.

${ }^{3 /}$ Ho: todos los miembros del panel son estacionarios. Ha: algunos miembros del panel contienen raíz unitaria.

${ }^{4 /}$ El estadístico P sigue una distribución Chi-cuadrado inversa.

* Se rechaza la Ho al 5\%. ** Se rechaza la Ho al $1 \%$.

Nota: se remueven los promedios (cross sectional) a las series en niveles.

Contraste de raíz unitaria: Intercepto y tendencia (series en niveles)

\begin{tabular}{|c|c|c|c|c|c|c|c|c|c|c|c|}
\hline & 30 días & & 90 días & & 180 día & & 360 día & & tasa dólar & & encaje \\
\hline Levin, Lin y Chu ${ }^{1 /}$ & $-9,9163$ & $* *$ & $-8,5248$ & $* *$ & $-6,7057$ & $* *$ & $-13,302$ & $* *$ & $-7,5677$ & $* *$ & 0,9478 \\
\hline Breitung $^{1 /}$ & $-5,6687$ & ** & $-4,7367$ & $* *$ & $-4,8797$ & $* *$ & $-7,1898$ & ** & $-11,6788$ & $* *$ & $-0,38$ \\
\hline Im, Pesaran y Shin ${ }^{2 /}$ & $-10,647$ & $* *$ & $-10,627$ & $* *$ & $-10,825$ & $* *$ & $-12,13$ & ** & $-14,5992$ & $* *$ & $-0,334$ \\
\hline Fisher-ADF $2 / 4 / 5 /$ & 58,8904 & & 42,7158 & $* *$ & 49,386 & $* *$ & 72,9373 & $* *$ & 104,8468 & $* *$ & 8,0228 \\
\hline Fisher-PP ${ }^{2 / 4 / 5 /}$ & 160,063 & $* *$ & 160,962 & $* *$ & 168,987 & $* *$ & 221,105 & ** & 338,1202 & $* *$ & 4,4525 \\
\hline $\mathrm{Hadri}^{3 /}$ & 3,953 & ** & 4,777 & ** & 3,6188 & $* *$ & 3,4822 & ** & 3,7263 & ** & 2,0675 \\
\hline
\end{tabular}

${ }^{1 /}$ Ho: los miembros del panel contienen raíz unitaria. Ha: los miembros del panel son estacionarios.

${ }^{2 /}$ Ho: todos los miembros del panel contienen raíz unitaria. Ha: algunos miembros del panel son estacionarios.

${ }^{3 /}$ Ho: todos los miembros del panel son estacionarios. Ha: algunos miembros del panel contienen raíz unitaria.

${ }^{4 /}$ El estadístico P sigue una distribución Chi-cuadrado inversa.

${ }^{5 /}$ Solo tendencia.

* Se rechaza la Ho al 5\%. ** Se rechaza la Ho al $1 \%$.

Nota: se remueven los promedios (cross sectional) a las series en niveles. 
Anexo 2. Tasa de interés de créditos comerciales en MN para distintos plazos

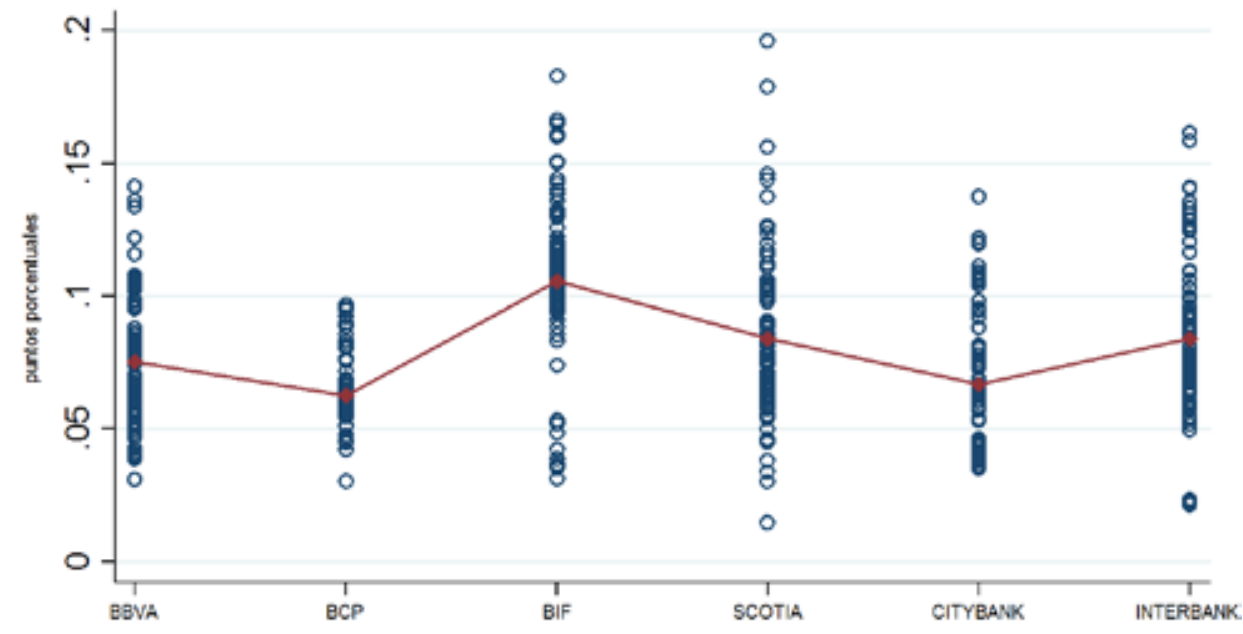

O tasa de interés comercial hasta 30 dias __ tasa de interés comercial hasta 30 dias - promedio

Fuente: SBS. Elaboración propia.

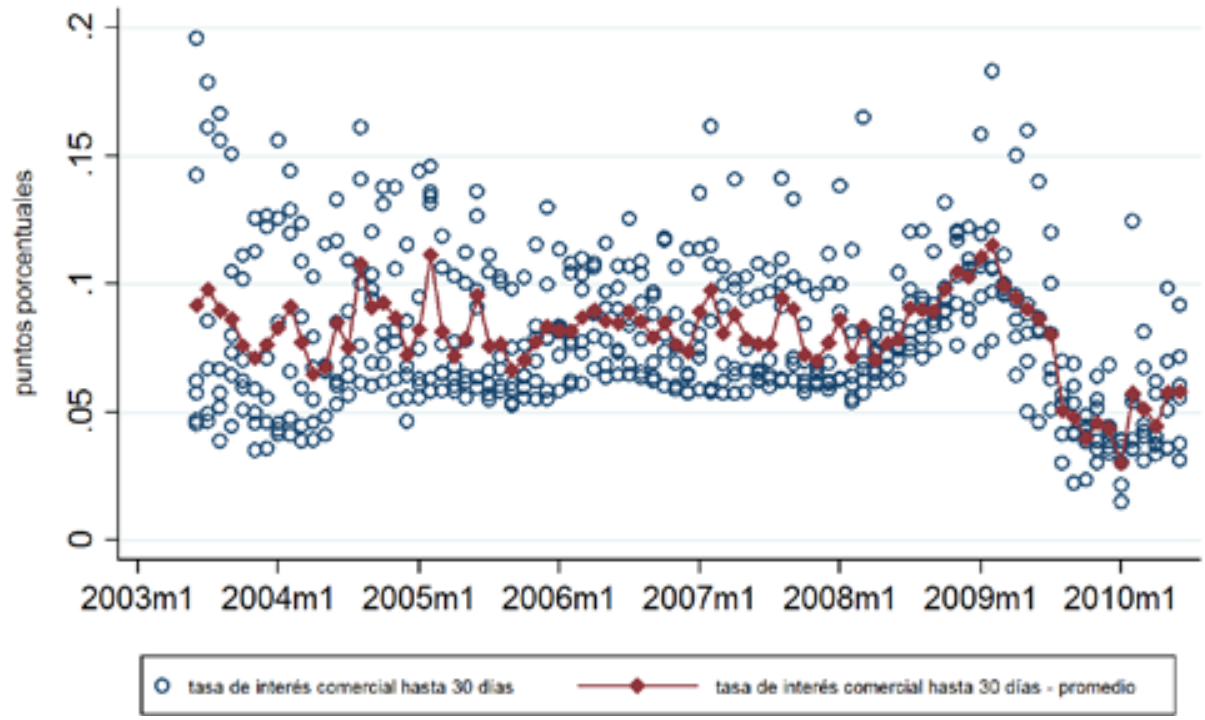

Fuente: SBS. Elaboración propia. 


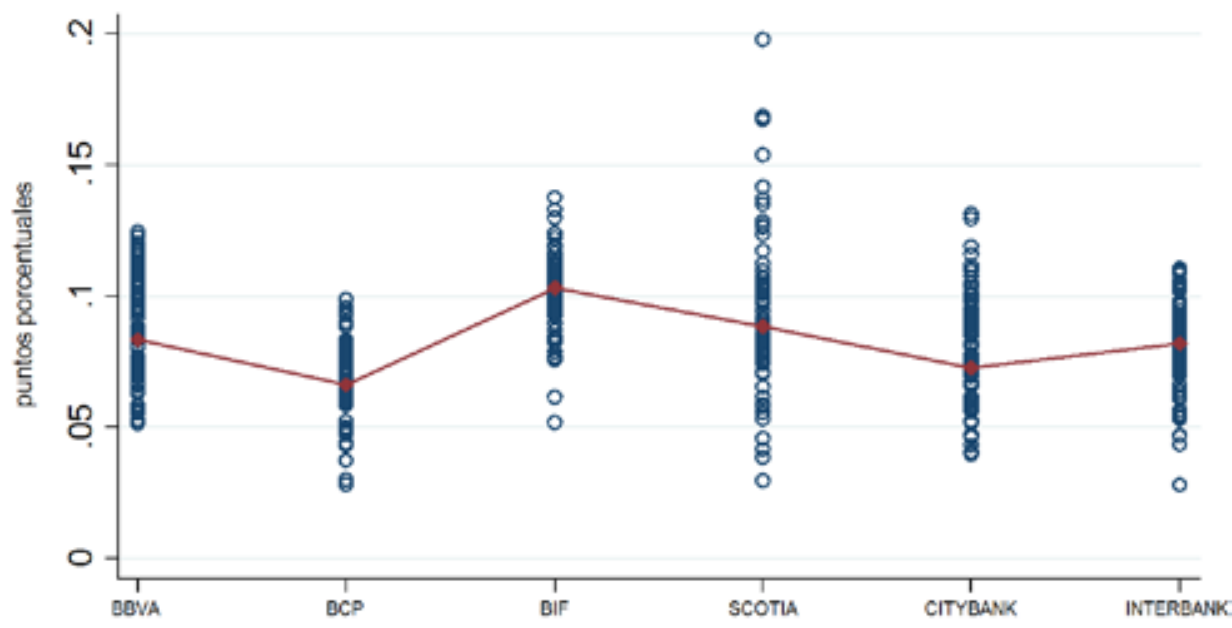

O tasa de interts comercial hasta 90 dias —— tasa de intert's comercial hasta 90 dias - promedio

Fuente: SBS. Elaboración propia.

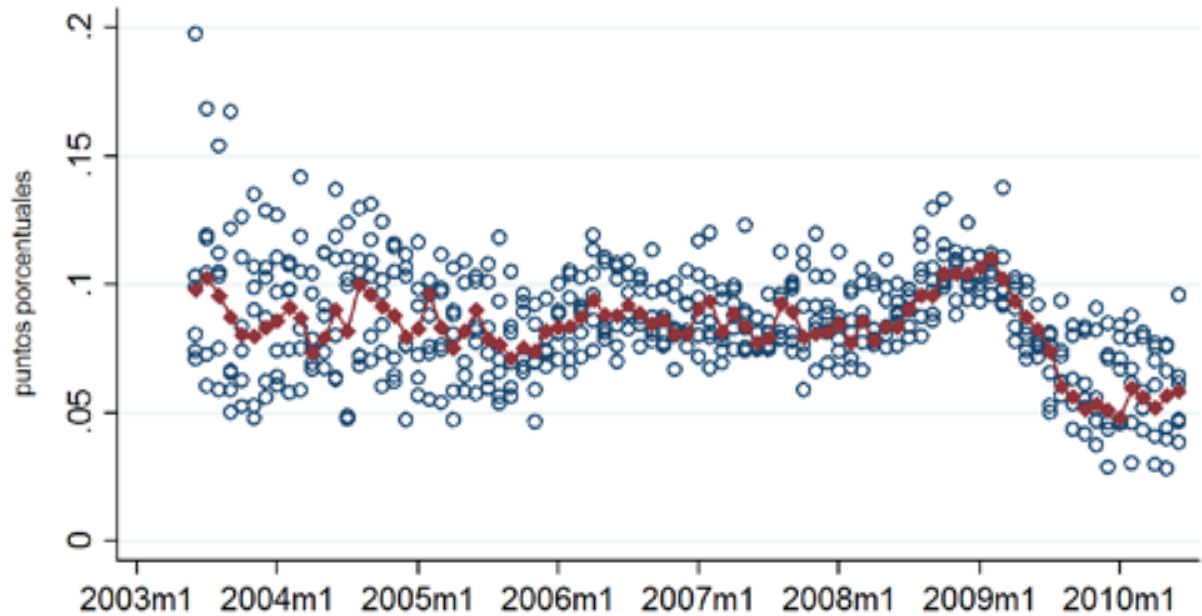

O tasa de interts comercial hasta 90 dias

tasa de interés comercial hasta 80 dias - promedic

Fuente: SBS. Elaboración propia. 


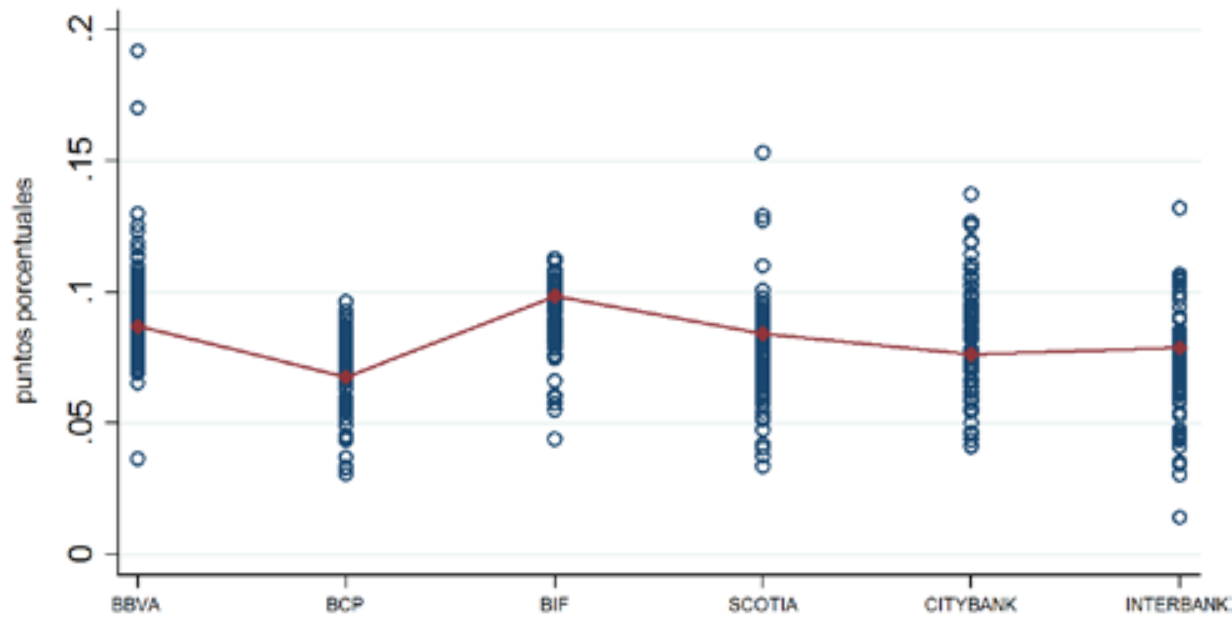

O tasa de interts comercial hasta 180 dlas

tasa de interés comercial hasta 190 dias - promedio

Fuente: SBS. Elaboración propia.

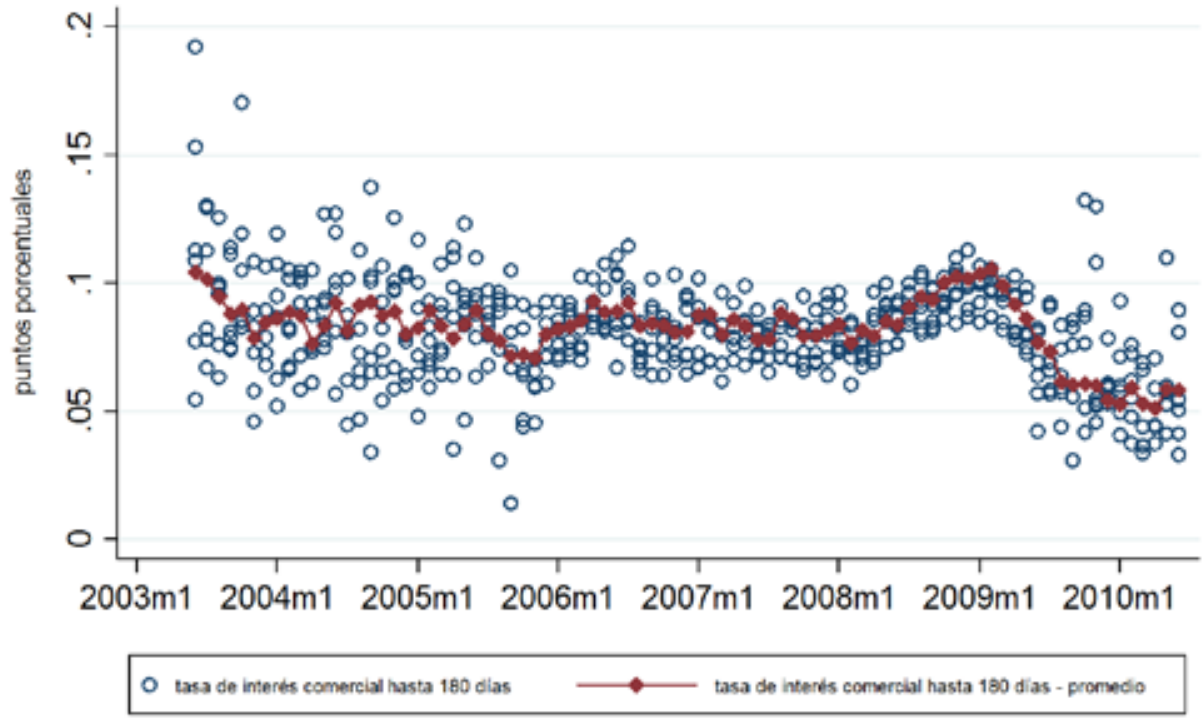

Fuente: SBS. Elaboración propia. 


\section{REFERENCIAS BIBLIOGRÁFICAS}

Armas, A., C. Castillo y M. Vega (2014). Inflation Targeting and Quantitative Tighteing: Effects of Reserve Requirements in Peru, Documentos de Trabajo 2014-003. Banco Central de Reserva del Perú.

Arellano, M. y S. Bond (1991). Some Tests of Specification for Panel Data: Monte Carlo evidence and an application to employment equations. Review of Economic Studies, 58(2), 277-297. https://doi.org/10.2307/2297968

Banco Central de Reserva del Perú (2009). Reporte Inflación. Junio.

Bernanke, B. y A. Blinder (1988). Is it Money or Credit, or both or neither? Credit, Money and Aggregate Demand. American Economic Review, 78(2), 435-439.

Bernanke, B. y M. Gertler (1995). Inside the black box: The Credit Channel of Monetary Policy Transmission. Journal of Economic Perspectives, 9(4), 27-48. https://doi.org/10.1257/jep. 9.4.27

Berstein, S. y J. R. Fuentes (2003). Is There Lending Rate Stickiness in the Chilean Banking Industry? Documento de Trabajo 218. Banco Central de Chile.

Dancourt, O. (2012). Crédito bancario, tasa de interés de politica y tasa de encaje en el Perú. Documento de trabajo 2012-342. Departamento de Economía de la Pontificia Universidad Católica del Perú.

Dancourt, O. (2014). Inflation Targeting in Peru: The Reasons for the Success. Documento de trabajo 2014-386. Departamento de Economía de la Pontificia Universidad Católica del Perú.

Ehrman, M., L. Gambacorta, J. Martinez-Pages, P. Sevestre y A. Worms (2001). Financial Systems and the Role of Banks in the Monetary Policy Transmission in the Euro Area. ECB Working Paper 105.

Gambacorta, L. (2004). How do Banks Set Interest Rates? NBER Working Paper 10295.

Heffernan, S. (1997). Modeling British Interest Rates Adjustment: An Error Correction Approach. Economica, 64, 211-231. https://doi.org/10.1111/1468-0335.00074

Judson, R. y A. Owen (1999). Estimating Dynamic Panel Data Models: A Guide for Macroeconomists. Economic Letters, 65, 9-15. https://doi.org/10.1016/S0165-1765(99)00130-5

Kashyap, A. K. y J. C. Stein (2000). What Do a Million Observations on Banks Say About the Transmission of Monetary Policy? American Economic Review, 90(3), 407-428. https:// doi.org/10.1257/aer.90.3.407

Lahura, Erick (2006). El efecto traspaso de la tasa de interés y la política monetaria en el Perú: 19952004. Revista Estudios Económicos 13. Banco Central de Reserva del Perú. Disponible en http://www.bcrp.gob.pe/docs/Publicaciones/Revista-Estudios-Economicos/13/EstudiosEconomicos-13-1.pdf

Stiglitz, J. y B. Greenwald (2003). Towards a New Paradigm in Monetary Economics. Cambridge: Cambridge University Press. https://doi.org/10.1017/CBO9780511615207

Takeda, M., F. Rocha y T. Nakane (2005). The Reaction of Bank Lending to Monetary Policy in Brazil. Revista Brasileira de Economí, 59(1), 107-126. https://doi.org/10.1590/S003471402005000100005

Vaugnet, V., M. Collin y E. Dhynne (2007). Monetary Policy and the Adjustment of Belgian Private Bank Interest Rates. An Econometric Analysis. National Bank of Belgium, Research Department.

Weth, M. A. (2002). The Pass-through from Market Interest Rates to Bank Lending Rates in Germany, Deutsche Bundesbank Discussion Paper, 11(02).

Documento recibido el 8 de octubre de 2016 y aprobado el 8 de noviembre de 2016 